\title{
BROOKHISEN
}

NATIONAL LABORATORY

BNL-94466-2010-CP

\section{Polarized proton beams in RHIC}

\begin{abstract}
A. Zelenski
Presented at the XX International Baldin Seminar on Relativistic Nuclear Physics and Quantum Chromodynamics

Dubna, Russia

October 4-9, 2010
\end{abstract}

Collider-Accelerator Department

\section{Brookhaven National Laboratory}

\section{U.S. Department of Energy DOE Office of Science}

Notice: This manuscript has been authored by employees of Brookhaven Science Associates, LLC under Contract No. DE-AC02-98CH10886 with the U.S. Department of Energy. The publisher by accepting the manuscript for publication acknowledges that the United States Government retains a non-exclusive, paid-up, irrevocable, world-wide license to publish or reproduce the published form of this manuscript, or allow others to do so, for United States Government purposes.

This preprint is intended for publication in a journal or proceedings. Since changes may be made before publication, it may not be cited or reproduced without the author's permission. 


\section{DISCLAIMER}

This report was prepared as an account of work sponsored by an agency of the United States Government. Neither the United States Government nor any agency thereof, nor any of their employees, nor any of their contractors, subcontractors, or their employees, makes any warranty, express or implied, or assumes any legal liability or responsibility for the accuracy, completeness, or any third party's use or the results of such use of any information, apparatus, product, or process disclosed, or represents that its use would not infringe privately owned rights. Reference herein to any specific commercial product, process, or service by trade name, trademark, manufacturer, or otherwise, does not necessarily constitute or imply its endorsement, recommendation, or favoring by the United States Government or any agency thereof or its contractors or subcontractors. The views and opinions of authors expressed herein do not necessarily state or reflect those of the United States Government or any agency thereof. 


\title{
POLARIZED PROTON BEAMS IN RHIC
}

\author{
A.Zelenski \\ Brookhaven National Laboratory, Upton, NY.
}

\begin{abstract}
The polarized beam for RHIC is produced in the optically-pumped polarized $\mathrm{H}^{-}$ion source and then accelerated in Linac to $200 \mathrm{MeV}$ for strip-injection to Booster and further accelerated $24.3 \mathrm{GeV}$ in AGS for injection in RHIC. In 2009 Run polarized protons was successfully accelerated to $250 \mathrm{GeV}$ beam energy. The beam polarization of about $60 \%$ at 100 $\mathrm{GeV}$ beam energy and $36-42 \%$ at $250 \mathrm{GeV}$ beam energy was measured with the $\mathrm{H}$-jet and pCarbon CNI polarimeters. The gluon contribution to the proton spin was studied in collisions of longitudinally polarized proton beams at $100 \times 100 \mathrm{GeV}$. At $250 \times 250 \mathrm{GeV}$ an intermediate boson $\mathrm{W}$ production with the longitudinally polarized beams was studied for the first time.
\end{abstract}

\section{Introduction.}

Collisions of protons at energies $\sqrt{\mathrm{S}}=200-500 \mathrm{GeV}$ and large transfer momentum $\left(\mathrm{p}_{\mathrm{T}}>10\right.$ $\mathrm{GeV} / \mathrm{c}$ ) are described as a parton collisions (quarks, gluons) and for polarized proton beams these partons are polarized too. The analyzing powers for polarized parton scattering can be directly calculated in the frame of perturbative QCD. This provides unique opportunity for the proton spin structure studies, fundamental tests of QCD predictions with possible extension to probe the physics beyond "Standard Model"/1,2 /. RHIC is the first collider where the "Siberian snake" technique was successfully implemented to avoid the resonance depolarization during beam acceleration in AGS and RHIC /3/ (see Fig.1). A luminosity of a $6010^{30} \mathrm{~cm}^{-2} \mathrm{sec}^{-1}$ (peak $120 \cdot 10^{30}$ ) for polarized proton collisions in RHIC at $\sqrt{ } \mathrm{S}=500 \mathrm{GeV}$ energy was produced by colliding 112 bunches in each ring at $1.4 \cdot 10^{11}$ protons/bunch intensity.

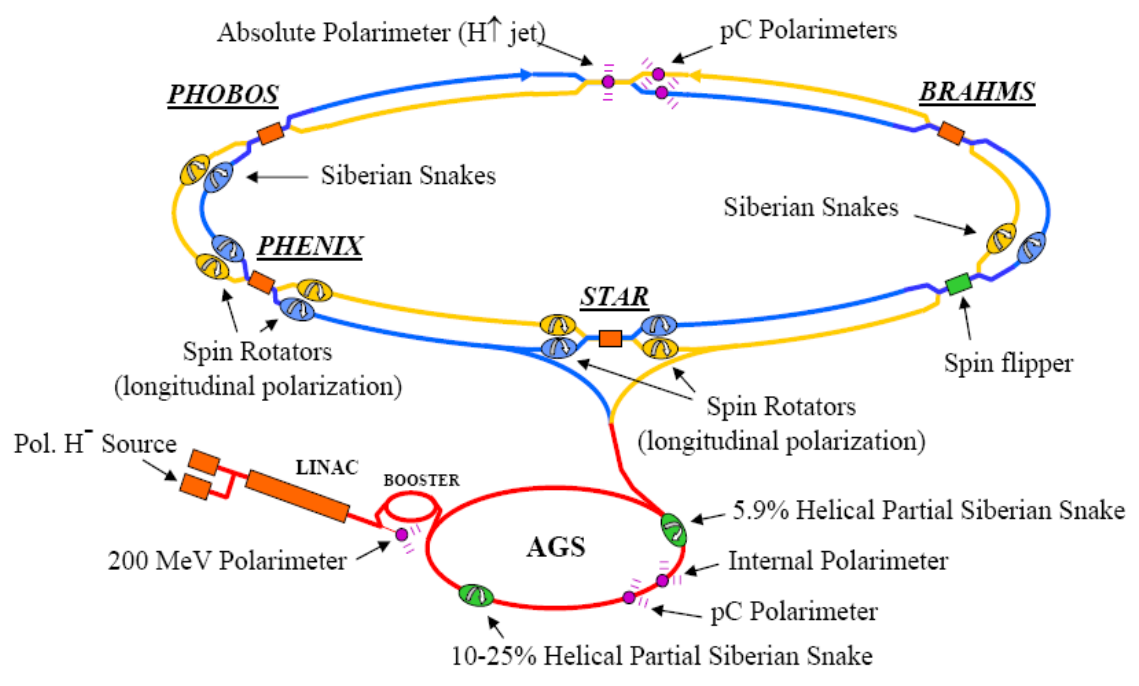

Fig.1. Polarization hardware layout of the RHIC Collider-Accelerator complex.

It is also for the first time the intensity of the polarized beams produced in an Optically Pumped Polarized $\mathrm{H}^{-}$Ion Source (OPPIS) was sufficient to charge RHIC to the maximum intensity limited by the beam-beam interaction. Polarimetry is another essential component of the polarized collider facility. A complete set of polarimeters includes: Lamb-shift polarimeter at 
the source energy, a $200 \mathrm{MeV}$ polarimeter after the linac, and polarimeters in AGS and RHIC based on proton-Carbon scattering in Coulomb-Nuclear Interference (CNI) region. A polarized hydrogen-jet polarimeter was used for the absolute polarization measurements in RHIC /4/.

Longitudinally polarized beams for the STAR and PHENIX experiments are produced with the spin rotators, which are tuned using "local polarimetrs" based on asymmetry in neutron production for $\mathrm{pp}$ collisions. The STAR and PHENIX detectors provide complimentary coverage of the different polarization processes.

\section{Polarized sources development at RHIC.}

Feasibility study of new techniques for the production of polarized electron, $\mathrm{H}^{-}$(proton), $\mathrm{D}^{-}$ (D+) and ${ }^{3} \mathrm{He}^{++}$ion beams are in progress at BNL. The OPPIS delivers beam for polarization studies in RHIC. The polarized deuteron beam will be required for the future deuteron Electron Dipole Moment (EDM) experiment, and the polarized electron (see I. Ben-Zvi talk in this Workshop) and ${ }^{3} \mathrm{He}^{++}$ion beam is a part of the experimental program for the future eRHIC (Electron- Ion) collider.

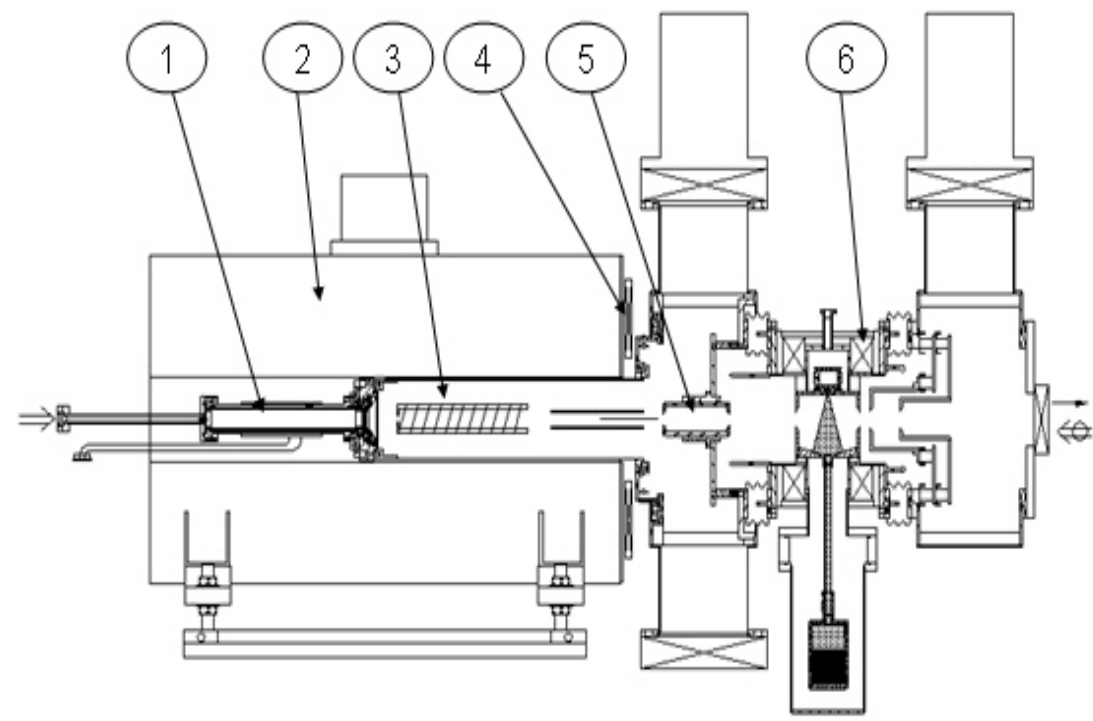

Fig.2. Layout of the RHIC OPPIS: 1) Electron -Cyclotron Resonance primary proton source:

2). Super-conducting Solenoid; 3) Optically-Pumped Rubidium Cell; 4). Correction coil; 5). Sona-shield; 6). Na-jet ionizer.

The present operational polarized source (OPPIS) for RHIC is based on spin-transfer proton (or atomic hydrogen) collisions in the optically-pumped Rb vapor cell. In the BNL OPPIS, an ECR-type source produces primary proton beam of a $2.0-3.0 \mathrm{keV}$ energy, which is converted to electron-spin polarized $\mathrm{H}$ atoms by electron pick-up in an optically pumped $\mathrm{Rb}$ vapor cell (see Fig.2). A pulsed Cr:LISAF laser of a $1 \mathrm{~kW}$ peak at 500 us pulse duration is used for optical pumping. Electrostatic deflection plates downstream of the polarized alkali remove any residual $\mathrm{H}^{+}$or other charged species. The electron-polarized $\mathrm{H}^{0}$ beam then passes through a magnetic field reversal region, where the polarization is transferred to the nucleus via the hyperfine interaction (Sona-transition). The nuclear polarized $\mathrm{H}$ atoms are then negatively ionized in a Najet vapor cell to form nuclear polarized $\mathrm{H}^{-}$ions. Alternatively, the $\mathrm{H}$ atoms can be ionized in a $\mathrm{He}$ gaseous cell to form polarized protons. After ionization polarized $\mathrm{H}^{-}$ions are accelerated from $3.0 \mathrm{keV}$ to $35 \mathrm{keV}$ energy by a negative $32 \mathrm{kV}$ pulsed voltage applied to the ionizer cell.

The OPPIS technique is a multi-step polarization-transfer process. At each step there is some loss of polarization. Detailed studies of polarization losses in the RHIC OPPIS and the source 
parameters optimization resulted in the OPPIS polarization increase to $86-90 \%$. The significant gain of about 5\% was obtained from experimental studies and numerical simulations of the Sona-transition efficiency. The simulations were done using code, which was developed at INR, Moscow/5/.

The OPPIS produces routinely 0.5-1.0 mA (maximum $1.6 \mathrm{~mA}$ ) $\mathrm{H}^{-}$ion current with $400 \mu \mathrm{s}$ pulse duration and 80-85\% polarization /6/. The beam is accelerated to $200 \mathrm{MeV}$ with an RFQ and linac for strip-injection to the Booster. About $60 \%$ of the OPPIS beam intensity can be accelerated to $200 \mathrm{MeV}$. The $400 \mu \mathrm{s} \mathrm{H}^{-}$ion pulse is captured in a single Booster bunch which contains about $4 \cdot 10^{11}$ polarized protons. Single bunch is accelerated in the Booster to $2.5 \mathrm{GeV}$ kinetic energy and then transferred to the AGS, where it is accelerated to $24.3 \mathrm{GeV}$ for injection to RHIC.

was moved from the $750 \mathrm{keV}$ line to the $35 \mathrm{keV}$ line to align the spin to the vertical direction before injection to the RFQ. The upgrade resulted in reduced spin precession, better optics matching between RFQ and Linac which reduced the emittance degradation in the Linac (in both transverse and longitudinal phase space). This smaller emittance was propagated through the accelerator chain, and resulted in smaller emittance and higher polarization in RHIC.

OPPIS upgrade with the Atomic Hydrogen Beam Injector. The ECR proton source is operated in high magnetic field. It has low hydrogen gas consumption, which makes possible a dc OPPIS operation with intensity in excess of $1.0 \mathrm{~mA}$. However, the proton beam produced in the ECR source has a comparatively low emission current density and high beam divergence. This limits further current increase and gives rise to inefficient use of the available laser power for optical pumping. In fact only about $15 \%$ of the electron-spin polarized hydrogen atoms produced in $\mathrm{Rb}$ cell is within the ionizer cell acceptance.

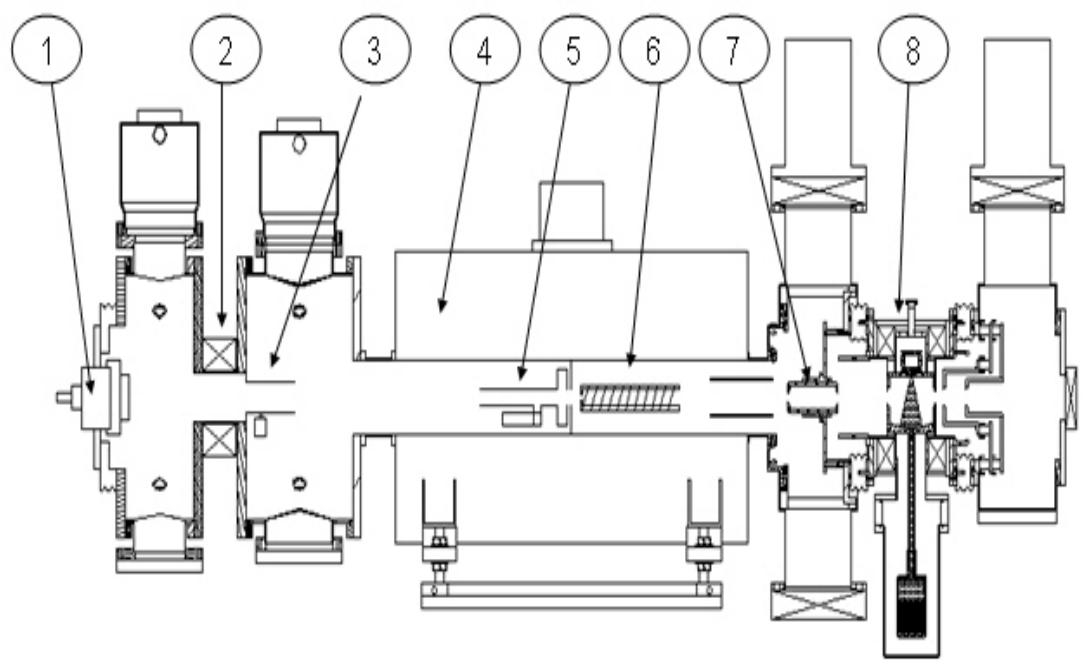

FIG.3. Layout of the OPPIS with atomic hydrogen injector: 1-high-brightness proton source; 2-focussing solenoid; 3-pulsed hydrogen neutralization cell;4- super conducting solenoid $30 \mathrm{kG}$; 5-Pulsed He ionizer cell; 6-opticallypumped $\mathrm{Rb}$ cell; 7-Sona shield; 8-sodium-jet ionizer cell.

In pulsed operation, suitable for application at high-energy accelerators and colliders, the ECR source limitations can be overcome by using instead of ECR a high brightness proton source outside the magnetic field $/ 7 /$. Following neutralization in hydrogen, the high brightness 5.0-8.0 keV atomic $\mathrm{H}^{0}$ beam is injected into a superconducting solenoid, where both a He ionizer cell and an optically-pumped $\mathrm{Rb}$ cell are situated in the same $25-30 \mathrm{kG}$ solenoid field, which is required to preserve the electron-spin polarization. The injected $\mathrm{H}$ atoms are ionized in the $\mathrm{He}$ cell with $80 \%$ efficiency to form a low emittance intense proton beam, which enters the polarized 
$\mathrm{Rb}$ vapor cell (see FIG.3). The protons pick up polarized electrons from the Rb atoms to become a beam of electron-spin polarized $\mathrm{H}$ atoms (similar to ECR based OPPIS). A negative bias of about 2.0-5.0 kV applied to the He cell decelerates the proton beam produced in the cell to the $3.0 \mathrm{keV}$ beam energy optimal for the charge-exchange collisions in the $\mathrm{Rb}$ and sodium cells. This also would allow the energy separation of the polarized hydrogen atoms produced after lower energy proton neutralization in $\mathrm{Rb}$ vapor and residual hydrogen atoms of the primary beam. Residual higher energy atoms will be neutralized with lower efficiency in Rb cell (due to cross-section decrease at higher energy) and unpolarized component will be further suppressed by lower $\mathrm{H}$ - ion yield at 5.0-8.0 $\mathrm{keV}$ atomic beam energy. The $\mathrm{H}^{-}$ion beam acceleration (by -32 $\mathrm{kV}$ pulsed voltage applied to the ionizer cell) will produce polarized $\mathrm{H}$ - ion beam of a $35 \mathrm{keV}$ beam energy and unpolarized beam of a $40-43 \mathrm{keV}$ beam energy. Further suppression of unpolarized higher energy ion beam can be done in the Low Energy Beam Transport Line (LEBT).

Atomic hydrogen beam current equivalent densities greater than $100 \mathrm{~mA} / \mathrm{cm}^{2}$ can be obtained at the $\mathrm{Na}$ jet ionizer location (about $200 \mathrm{~cm}$ from the source) by using a very high brightness fast atomic beam source developed at BINP, Novosibirsk. This was tested in experiments at TRIUMF, where more than $10 \mathrm{~mA}$ polarized $\mathrm{H}^{-}$and $50 \mathrm{~mA}$ proton beam intensity was demonstrated $/ 6 /$. Higher polarization is also expected with the fast atomic beam source due to: a) elimination of neutralization in residual hydrogen; b) better Sona-transition transition efficiency for the smaller $\sim 1.5 \mathrm{~cm}$ diameter beam; c) use of higher ionizer field (up to $3.0 \mathrm{kG}$ ), while still keeping the beam emittance below $2.0 \pi \mathrm{mm} \cdot \mathrm{mrad}$, because of the smaller beam -1.5 $\mathrm{cm}$ diameter. All these factors combined will further increase polarization in the pulsed OPPIS to $\sim 90 \%$ and the source intensity to over $10 \mathrm{~mA}$.

The RHIC polarized source upgrade for higher intensity and polarization is approved and fully funded for implementation in 2009-12. The source will provide the high intensity low emittance (high brightness) beam for polarized RHIC luminosity upgrade and for future eRHIC facilities.

Proposal for polarized ${ }^{3} \mathrm{He}^{++}$source for eRHIC. Polarized neutrons collisions can be studied with the deuteron beams. Unfortunately due to small deuteron anomalous magnetic moment G-factor $(\mathrm{G}=-0.143)$ the "Siberian snakes" and spin rotators will be too weak to be effective for the polarized deuteron acceleration in AGS and RHIC. Polarized beams of ${ }^{3} \mathrm{He}^{++}$ ions also contain the polarized neutron component (as a deuteron) and its $\mathrm{G}$-factor: $\mathrm{G}=-4.184$ is even larger than the proton $G_{p}$ value, therefore the AGS and RHIC Siberian snake can be operated at lower field to preserve polarization during acceleration ${ }^{17}$. In this case the difficulty is the polarized ${ }^{3} \mathrm{He}^{++}$source. The proposed polarized ${ }^{3} \mathrm{He}^{++}$acceleration in RHIC (and also for future RHIC upgrade to electron ion collider eRHIC) will require about $2 \cdot 10^{11}$ ions in the source pulse and about $10^{11}$ ions in the RHIC bunch. To deliver these ions in a 20 us pulse duration for injection to Booster, the source peak current has to be about $1000 \mu \mathrm{A}$, which is 10000 times higher than ever achieved in any ${ }^{3} \mathrm{He}^{++}$ion sources. A new technique has been proposed for production of high intensity ${ }^{3} \mathrm{He}^{++}$ion beam. It is based on ionization of nuclear polarized ${ }^{3} \mathrm{He}$ gas in the Electron Beam Ion Source (EBIS) /8/ ( see FIG.4). The highest ${ }^{3} \mathrm{He}$ nuclear polarization in excess of $80 \%$ was achieved so far by the metastability exchange technique. In this method, ${ }^{3} \mathrm{He}$ gas at typically 1 torr pressure is contained in a glass bulb and a weak RF discharge is maintained in the gas. Metastable atoms in the $2{ }^{3} \mathrm{~S}_{1}$ state are produced in the discharge and may be polarized by means of optical pumping with circularly polarized $\left(2{ }^{3} \mathrm{~S}_{1}\right.$ $\left.2^{3} \mathrm{P}_{0}\right) 1083 \mathrm{~nm}$ light. The polarization is transferred to the ground state atoms by the metastability-exchange collisions. In the cesium coated quartz cell the long $(>100 \mathrm{~h})$ polarization relaxation time was obtained resulting in high ground state polarization. In the proposed technique, the polarized ${ }^{3} \mathrm{He}$ atoms consumption for injection to an ionizer (another polarized atoms loss factor) is very small, of the order of $10^{13}-10^{14} \mathrm{He}$ atoms $\mathrm{sec}^{-1}$ and high polarization is expected. 


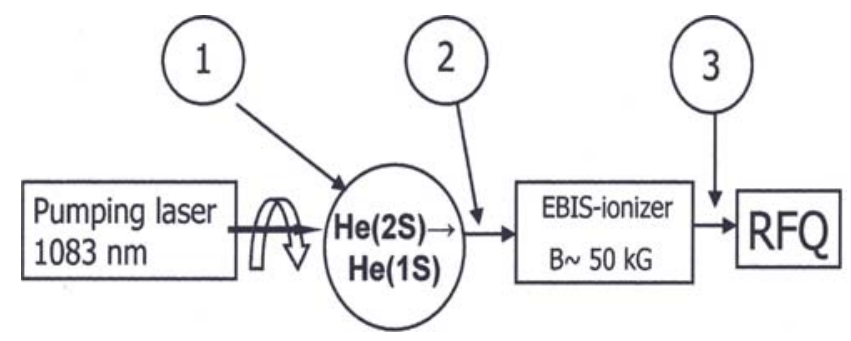

Fig.4. Schematic diagram of the polarized ${ }^{3} \mathrm{He}^{++}$ion source. 1-metastability-exchange polarizing cell; $2-{ }^{3} \mathrm{He}$ transfer line; $3 \cdot 10^{12}$ polarized ${ }^{3} \mathrm{He}^{++}$ions for injection to RFQ.

An EBIS is under construction at BNL as an alternative to the Tandem heavy ion injector for RHIC. It is proposed to use the EBIS to produce ${ }^{3} \mathrm{He}^{++}$by ionization of the polarized ${ }^{3} \mathrm{He}$ gas, which is fed from the polarizing cell. The ionization in the EBIS is produced in a $50 \mathrm{kG}$ magnetic field, which preserves the nuclear ${ }^{3} \mathrm{He}$ polarization while in the intermediate singlecharged ${ }^{3} \mathrm{He}^{+}$state. The ionization efficiency to the double-charged ${ }^{3} \mathrm{He}^{++}$will be close to $100 \%$ and the number of ions is limited to the maximum charge, which can be confined in the EBIS. From experiments with $\mathrm{Au}^{32+}$ ion production, one expects about $2.5 \cdot 10^{11}{ }^{3} \mathrm{He}^{++}$ions/pulse to be produced and extracted for subsequent acceleration and injection to RHIC.

After ${ }^{3} \mathrm{He}^{++}$acceleration to a few MeV/nucleon He-D or He-Carbon collisions can be used for polarization measurements. The Lamb-shift polarimeter at the source energy of $10-20 \mathrm{keV}$ can be used in the feasibility studies (similar to the OPPIS polarimeter). In this technique ${ }^{3} \mathrm{He}^{++}$ions are partially converted to $\mathrm{He}^{+}(2 \mathrm{~S})$ - metastable ions in the alkali vapor cell. Then the hyperfine sublevel populations can be analyzed in the spin-filter device to extract the primary ${ }^{3} \mathrm{He}^{++}$ nuclear polarization.

A study of limitation on the maximum attainable nuclear polarization in the metastability exchange technique (at the very low polarized ${ }^{3} \mathrm{He}$ gas consumption rate) will be required to define the maximum attainable polarization. Possible depolarization effects during polarized ${ }^{3} \mathrm{He}$ gas injection to existing EBIS prototype and multi-step ionization process should be also studied. The expected ${ }^{3} \mathrm{He}^{++}$ion beam intensity is in excess of $2 \cdot 10^{11}$ ions/pulse with polarization in excess of $70 \%$.

\section{Polarized beam acceleration in AGS and RHIC.}

Polarized beams in AGS. Two partial Siberian snakes were installed in AGS to preserve polarization during beam acceleration from Booster energy $2.4 \mathrm{GeV}$ to $24 \mathrm{GeV}$ (see T.Roser talk at PSTP $2007 / 9 /$ ). With this snake configuration the polarization losses at all imperfection and vertical intrinsic resonances was eliminated except a few vertical intrinsic resonances near injection energy. As a result a polarized proton beam with $1.5 \cdot 10^{11}$ intensity/bunch and $65 \%$ polarization has been delivered for injection to RHIC in 2006-09 Runs. A spin tracking simulations showed that the remaining polarization losses $\sim 15 \%$ (beam polarization at injection to AGS was about $80-85 \%$ ) can be caused by: the loss due to horizontal intrinsic resonances along the ramp; loss associated with snake resonances near the strongest $36+\mathrm{v}$ : the loss from vertical intrinsic resonances near injection. All these losses can be reduced by the smaller beam emittance. In 2009 Run smaller beam emittance out of Linac, shorter strip-injection time to Booster (see above) and better beam injection matching from Booster to AGS resulted in smaller beam emittance in AGS and RHIC. Also a new lattice with the vertical tune in the spin tune gap near injection has been used to reduce the loss near injection energy. As a result $65-70 \%$ polarization was consistently delivered for injection to RHIC (see Fig.5). A new pulsed quadrupoles were installed in AGS to produce fast tune jump during the energy rump in attempt to eliminate all residual depolarization at the horizontal spin resonances $/ 10 /$. 


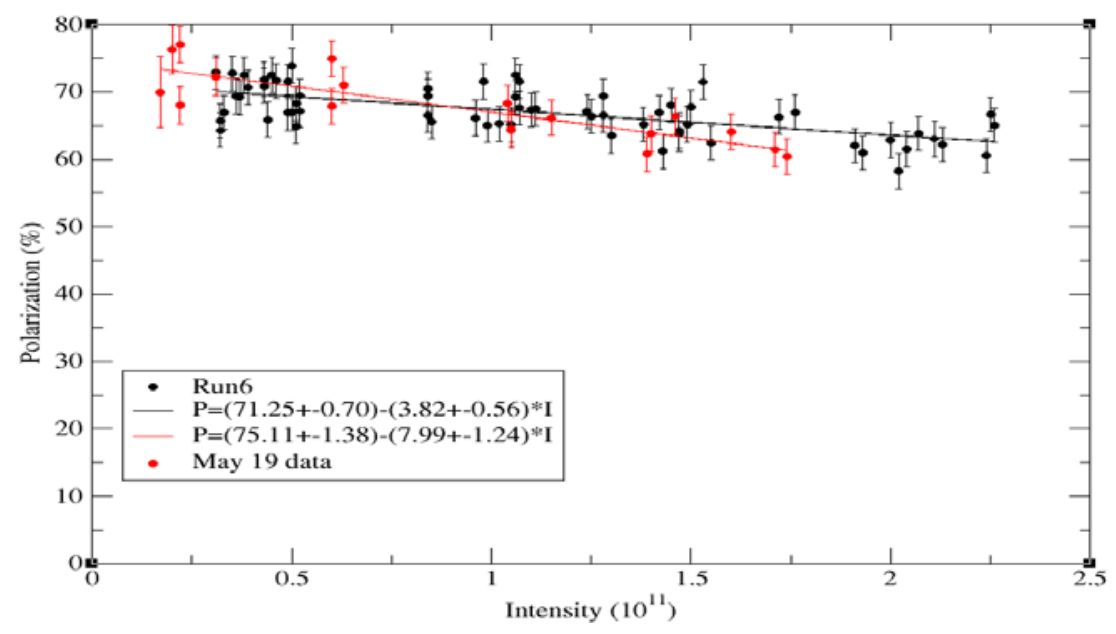

Fig.5. Polarization vs. bunch intensity in AGS.

Polarized beams in RHIC. The RHIC-heavy ion collider is the first high-energy machine where polarized proton acceleration was included in the primary design. The RHIC "full Siberian snake" (which rotates spin direction for $180^{\circ}$ ) is a superconducting helical magnet system of about $10 \mathrm{~m}$ long. Two $90^{\circ}$ helical spin rotators in each ring produce the longitudinal polarization for experiments in STAR and PHENIX detectors.

Up to 120 beam bunches can be accelerated and stored in each ring. The polarization direction of every RHIC bunch is determined by the spin-flip control system in the polarized ion source. Every single source pulse is accelerated and becomes the RHIC bunch of the requested polarity. By loading selected patters of spin direction sequences in the rings (such as: +-+- in one ring and +--+ in another) the experiments have all possible spin directions combinations for colliding bunches which greatly enhance the systematic error control.

Since 2005, RHIC has successfully accelerated polarized protons up to $100 \mathrm{GeV}$ with no polarization loss by carefully controlling the betatron tunes and the vertical orbit distortion. A record polarization of $60 \%$ was reached during the RHIC polarized proton operation in 2006 and routinely delivered in Run 9 . However, between $100 \mathrm{GeV}$ and $250 \mathrm{GeV}$, there are three strong intrinsic spin resonances which may cause polarization loss. These resonances are more than a factor of two stronger than the strong spin resonances below $100 \mathrm{GeV}$. Since the stronger the intrinsic resonance, the stronger the derived snake resonances, the tolerance on the nearby imperfection resonance, i.e. the vertical closed orbit distortion, is tighter. The numerical simulation shows that the imperfection resonance strength should be below 0.075 to avoid depolarization at these three strong intrinsic resonances $/ 11 /$. This corresponds to a closed orbit distortion of $0.3 \mathrm{~mm}$ rms value. These accuracies of the orbit and tune control were achieved in 2009 Run. As a result the proton polarization transfer efficiency (from injection to store) was improved to about $80 \%$ and on average about $42 \%$ polarization was measured during the 250 GeV physics stores.

A maximum polarized beam luminosity of a $45 \cdot 10^{30} \mathrm{~cm}^{-2} \mathrm{sec}^{-1}$ (about $30 \cdot 10^{30} \mathrm{~cm}^{-2} \mathrm{sec}^{-1}$ averaged over $8 \mathrm{hrs}$ store time) was obtained with 109 bunches of $1.6 \cdot 10^{11}$ bunch intensity in 2009 run.

\section{Polarization measurements in AGS and RHIC.}

Proton polarization measurements in the AGS and RHIC are based on proton-carbon and proton-proton elastic scattering in the Coulomb Nuclear Interference (CNI) region /12/. This 
process has a large cross-section and sizable analyzing power of a few percents which is expected to have weak energy dependence in the $24-250 \mathrm{GeV}$ energy range. A very thin (5 $\mu \mathrm{g} / \mathrm{cm} 2,5-10 \mu \mathrm{m}$ wide) carbon strip target in the high intensity circulating beam produces high collision rate and a highly efficient DAQ system acquires up to $5 \cdot 10^{6}$ carbon events/sec. High recoil carbon nuclei intensity from the scattering of the circulated proton beam in the thin carbon target is efficiently utilized in the silicon strip detectors and data acquisition system, which is capable to analyze the event rate up to a few millions/second. This gives unique possibilities for the fast, practically non-destructive polarization measurements. The polarization measurements during the beam energy ramp were also implemented in AGS and RHIC, which provides an insight of polarization losses pattern. Polarimeter operation in the scanning mode also gives polarization profile and beam profile (beam emittances including bunch by bunch measurements).

The CNI polarimeters were upgraded for the 2009 run. Two identical target motion mechanisms and detectors assemblies were installed in new vacuum chambers in each ring. One polarimeter was used for the vertical polarization and intensity profile measurements and the other for the horizontal profile measurements (or vice versa). As a result the systematic polarization, polarization profiles and emittance measurements were obtained for both transverse planes. This is in contrast to previous runs where measurements were limited to one plane due to long target switch times.

The absolute beam polarization at $100 \mathrm{GeV}$ beam energy was measured with a polarized $\mathrm{H}$-jet polarimeter $/ 4,13 /$. The simultaneous measurements in $\mathrm{p}$-Carbon and $\mathrm{H}$-jet polarimeters provide the calibration for $\mathrm{p}$-Carbon analyzing power. Fast $\mathrm{pC}$ polarimeter measures possible polarization losses during the energy ramp and possible polarization decay during the RHIC store.

\section{Summary}

The RHIC spin program is a great beneficiary of the latest development in the polarized ion source and polarized target technology. For the first time the polarized proton beam intensity in the high-energy accelerator is not limited by the polarized source intensity. In 2009 Run polarized protons was successfully accelerated to $250 \mathrm{GeV}$ beam energy. The beam polarization of a $60 \%$ at $100 \mathrm{GeV}$ beam energy and $42 \%$ at $250 \mathrm{GeV}$ beam energy was measured with the polarized $\mathrm{H}$-jet and p-Carbon CNI polarimeters. The gluon contribution to the proton spin was studied in collisions of longitudinally polarized proton beams at $\sqrt{S}=200 \mathrm{GeV}$. At $\sqrt{S}=500 \mathrm{GeV}$ an intermediate boson $\mathrm{W}$ production with the longitudinally polarized beams was studied for the first time.

\section{References.}

1. G. Bunce et al., "Prospects for Spin Physics in RHIC", Ann. Rev. Nucl. Part. Sci., 50, p. 525, (2000).

2. N. Saito et al., Proc. $16^{\text {th }}$ Int. Spin physics Symposium, p.58, World Scientific, (2005).

3. I. Alekseev et al., NIM A499, p.392, (2003).

4. A. Zelenski et al., NIM A536, p.248, (2005).

5. E. Antishev, A. Belov, AIP Conf.Proc.980, p.263,(2008).

6. A. Zelenski et al., AIP Conf. Proc. 570, p.179, (2000).

7. A. Zelenski et al., NIM A245, p.223, (1986).

8. A. Zelenski, J. Alessi, ICFA Beam Dynamics Newsletter, 30, p.39,(2003).

9. T. Roser, AIP Conf. Proc, 980, p.15, (2008).

10. H. Huang et al., "AGS Polarized Proton operation in Run 2009”, BNL-81823-2009-CP.

11. M. Bai et al., Proc. PAC2007, p.745, (2007)

12. I. Nakagava et al., AIP Conf. Proc, 980, p.380, (2008).

13. H. Okada et al., Phys. Letters, B638, p.450, (2006). 